\title{
The online laboratories for OCRA - Outreach Cosmic Ray Activities INFN project
}

\author{
C. Aramo ${ }^{a}{ }^{*}$, R. Antolini ${ }^{b}$, V. Boccic ${ }^{c}$, M. Buscemi $^{d}$, L. Caccianiga ${ }^{e}$, A. Candela ${ }^{b}$, G. \\ Cataldif, R. Colalillo ${ }^{a, g}$, F. Convenga ${ }^{f, h}$, M.R. Coluccia ${ }^{f}$, F. de Palma ${ }^{f, h}$, P. Di Nezza ${ }^{i}$, \\ G. Di Sciascio', C. Evoli ${ }^{b, k}$, G. Galbato ${ }^{c, l}$, S. Hemmer ${ }^{m}$, A. Giampaoli ${ }^{b}$, F. \\ lacoangeli $^{c}$, D. Liguori ${ }^{n}$, S. Miozzi ${ }^{j}$, R. Paolettio , N. Rossi ${ }^{b}$, D. Rozza ${ }^{p, q}$, M.
}

Schioppa ${ }^{n}$, I. Veronesi ${ }^{a, r}$ on behalf of the OCRA Collaboration

(a complete list of authors can be found at the end of the proceedings)

${ }^{a}$ INFN Sezione di Napoli, Napoli, Italy

${ }^{b}$ INFN Laboratori Nazionali del Gran Sasso, Assergi, Italy

${ }^{c}$ INFN Sezione di Roma I, Roma, Italy

${ }^{d}$ INFN Sezione di Catania, Catania, Italy

e INFN Sezione di Milano, Milano, Italy

${ }^{f}$ INFN Sezione di Lecce, Lecce, Italy

g Università di Napoli "Federico II", Dipartimento di Fisica "Ettore Pancini", Napoli, Italy

${ }^{h}$ Università del Salento, Dipartimento di Matematica e Fisica "Ennio de Giorgi"

${ }^{i}$ INFN Laboratori Nazionali di Frascati, Frascati, Italy

j INFN Sezione di Roma Tor Vergata, Roma, Italy

${ }^{k}$ Gran Sasso Science Institute, L'Aquila, Italy

$l$ Università La Sapienza, Dipartimento di Fisica, Roma, Italy

m INFN Sezione di Padova, Padua, Italy

${ }^{n}$ INFN Laboratori Nazionali di Frascati, Gruppo Collegato di Cosenza, Cosenza, Italy

o INFN Sezione di Pisa, Pisa, Italy

${ }^{p}$ INFN Laboratori Nazionali del Sud, Catania, Italy

${ }^{q}$ Dipartimento di Chimica e Farmacia, University of Sassari, Sassari, Italy

${ }^{r}$ Università di Salerno, Dipartimento di Matematica, Salerno, Italy

E-mail: carla.aramo@na.infn.it

"Presenter

(c) Copyright owned by the author(s) under the terms of the Creative Commons 
OCRA - Outreach Cosmic Ray Activities was born in 2018 as a national outreach project of INFN with the aim of collecting, within a national framework, the numerous public engagement activities in the field of cosmic ray physics already present at a local level in the divisions and laboratories. Since spring 2020 OCRA offers also a series of online laboratories on its website https://web.infn.it/OCRA/, designed not only to be used by students individually but also to be offered in the classroom by teachers. The cosmic ray path, present on the website, will be presented together with the online laboratories on the measurement of muons, from the one related to the dependence on the zenith angle made during the International Cosmic Day up to measurements of the flux dependency on the altitude in the atmosphere and in the water. Also, a laboratory allowing to analyze public data of the Pierre Auger Observatory will be presented. In addition, some teaching methods included in the "Teachers' area" of the OCRA website will be described. The developed cosmic ray path was also used to organize an online course for teachers of Italian high schools with the purpose of accompanying teachers when approaching the subject for the first time. About 70 teachers participated for a total of 9 lessons.

37th International Cosmic Ray Conference (ICRC 2021)

July 12 th - 23rd, 2021

Online-Berlin, Germany 


\section{Introduction}

The cosmic radiation, invisible to human eyes, encompasses all the fundamental issues of modern physics: from the origins and evolution of the Universe to the current composition of known forces and the intimate structure of matter. The study of this radiation has given rise to all modern theories and knowledge of the world around us.

On the other hand, cosmic radiation is easily intercepted and can easily "be made visible" through simple particle detector telescopes. This results in an excellent educational laboratory for introducing non-experts, especially students, to the study of physics and fundamental natural phenomena. The study of cosmic radiation is also closely linked to the other major themes of modern astrophysics, from dark matter to gravitational waves and neutrino oscillations and much more. Talking about cosmic rays therefore means talking about the exploration of the Universe in all its forms.

OCRA - Outreach Cosmic Ray Activities [1] founded in 2018 within the National Institute of Nuclear Physics (INFN), aims to collect the many public engagement activities in the field of cosmic ray physics already present locally and to disseminate them nationally (see the contribution by S. Hemmer in this conference). This article will describe, in particular, the new national online activities developed since spring 2020 and dedicated to high school students and teachers.

\section{The cosmic ray pathway}

During the spring 2020 lockdown, all OCRA activities were cancelled, and the need was felt to develop tools that could be used remotely, also to help many Italian students who needed to take their final year exams in high school. For this reason, it was decided to use the instrumentation and the experimental data to create an online pathway that would allow students to acquire the notions useful to understand what cosmic rays, extensive air showers (EAS), muons and other quantities useful to understand cosmic radiation are, as well as the instruments to detect them, especially to perform measurements of atmospheric muons under different conditions. This gave rise to the "cosmic rays" pathway on the OCRA website [2], which will be described in this article, and which also made it possible to create the OCRA course for high school teachers presented in January 2021 [3] and carried out in 9 meetings in spring for high school teachers.

\section{The online laboratories}

The laboratories are set up with simple devices for the detection of cosmic muons and describe their behaviour. Each one is dedicated to an experimental measurement and with the data provided you are guided through the analysis that allows the measurement of a physical quantity of muons, such as:

- measurement of the cosmic muon rate and track reconstruction

- measurement of muon angular distribution

- measurement of muon intensity as a function of zenith angle

- measurement of muons as a function of height in the atmosphere

- measurement of muons as a function of depth in water.

Some international experiments, such as the Pierre Auger Observatory [4], have made public part of their data, which can be downloaded directly from the official websites of the collaborations. So, Python scripts have also been developed to guide the students in exploring these data and analysing them, and to compare the results with the official ones published by the collaboration. In the following, the seven laboratories will be briefly presented.

Finally, OCRA also offers a "Teachers' Area", i.e. a short selection of materials to support the development of a teaching project in the classroom. 


\subsection{Measurement of the cosmic muon rate and track reconstruction}

At the INFN National Laboratories of Gran Sasso (LNGS) a cosmic ray telescope has been designed and built for use in public and educational events. The instrument, called "Cosmic Ray Cube" (CRC) [5], thanks to its compact structure of 4 double-plane horizontal scintillation levels orthogonal to each other, and the possibility of being battery powered, is easy to carry and allows to measure the flow of particles at various altitudes, their angular distribution, the efficiency of the detector for the variation of some operating parameters.

The telescope is capable of detecting the most penetrating component of secondary cosmic rays, the muons. Detection of the passage of muons within the CRC is done through the use of a technology that employs three types of detectors: plastic scintillators, Wave Length Shifter (WLS), silicon photomultipliers (SiPM) able to convert the light collected by WLS into an electrical signal easily digitizable. The CRC is also equipped with LEDs that light up when the particles pass, allowing the naked eye to follow the trajectory (Fig.1a)

The App "Cosmic Rays Live" has been developed downloadable from any smartphone, on which entire sets of data collected by the CRC can be saved (Fig. 1b). With Android or iOS system the data are automatically saved on the phone every time you connect to one of the sites where the telescopes are located. The data can then be analyzed to reconstruct the tracks left by muons in the telescope. In the webpage [5] you can follow, step by step, all the reconstruction of the trajectories as well as download an Excel file with the data and the App.
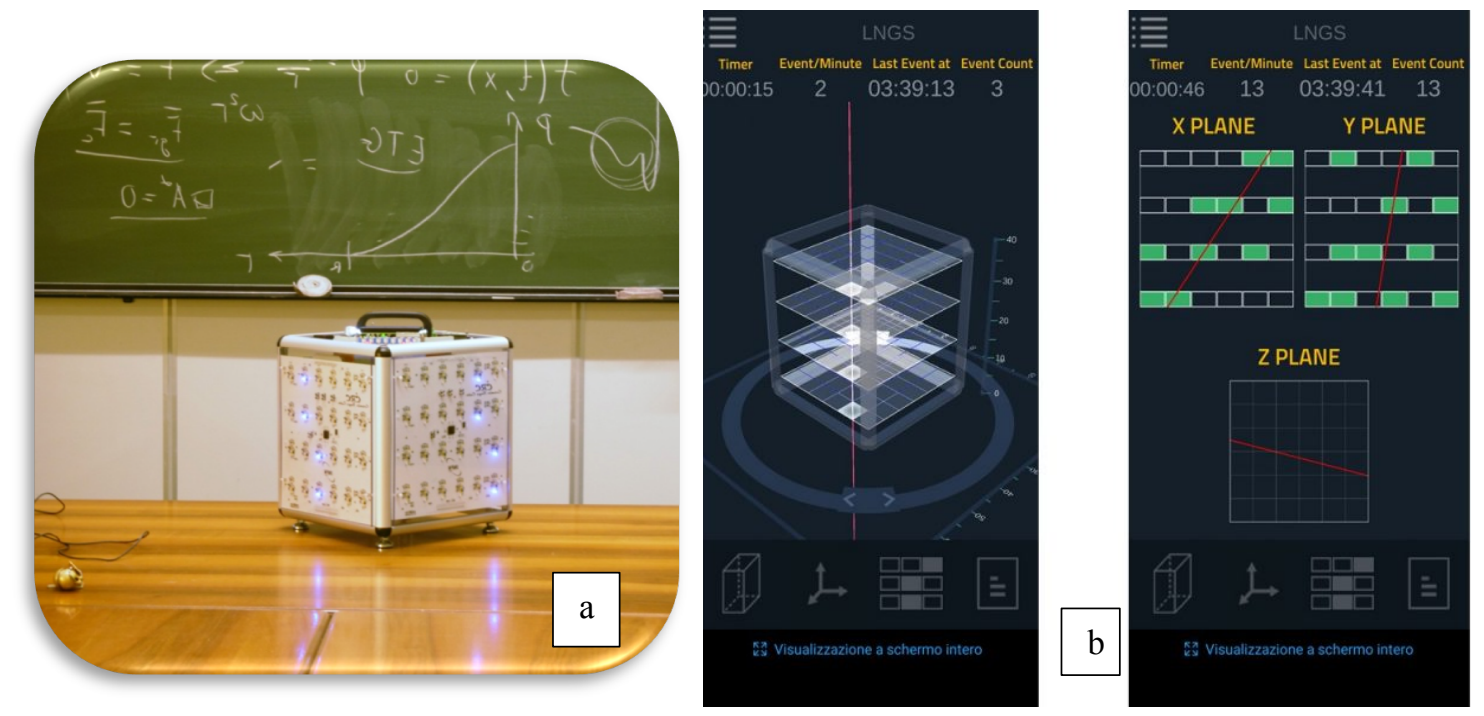

Figure 1: (a) Cosmic Ray Cube and (b) its App "Cosmic Rays Live".

\subsection{Measurement of muon angular distribution}

This laboratory allows the discovery of the detector installed in 2014 in the Toledo metro station in Naples (Fig. 2a) [6]. This telescope is similar to the CRC described in the previous paragraph but larger: it consists of 10 floors compared to the 4 of the CRC.

When a muon passes through the telescope, a light track is displayed as in Fig. 2a. The telescope is coupled to a multimedia touchscreen that allows the acquisition of data to reconstruct the direction of the muons and the display of their tracks on the screen (Fig. 2b).

In the tutorial [7], the reconstruction of the muon trajectory is described step by step, and two exercises are developed with Python Google Colab scripts. By this, it is possible to draw histograms of the distributions of the muon arrival directions, as well as to find which of the proposed curves best fits the distributions found by the fit procedure, and to choose between two different polynomial functions, the Gaussian or the asymmetric Gaussian. 

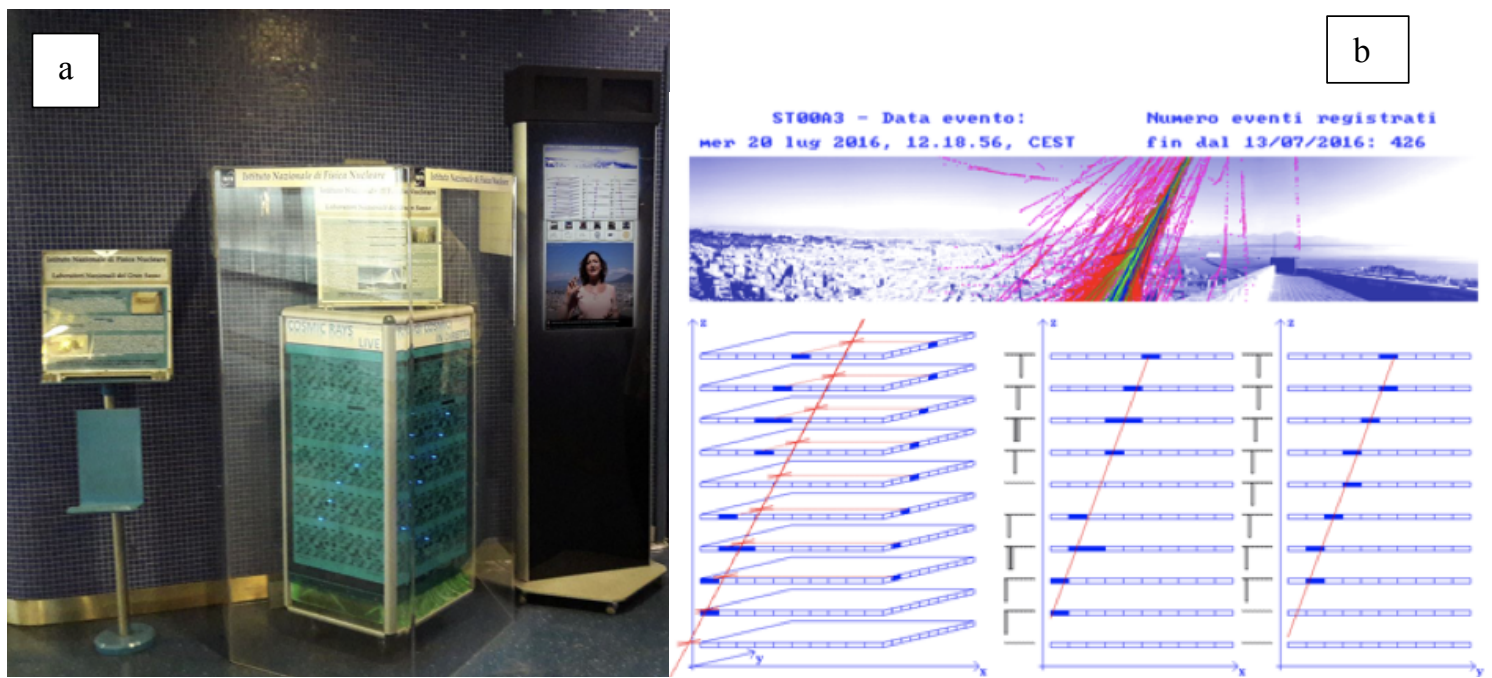

Figure 2: (a) Toledo telescope and (b) reconstruction of a muon track.

\subsection{Measurement of muon intensity as a function of Zenith angle}

The measurements described in this laboratory [8] were carried out during the International Cosmic Day (ICD) [9], the day dedicated to cosmic rays that allows students to get in touch with research activity in physics, carry out a small experiment on cosmic rays, analyze the data, compare their results with other related groups from other universities or research institutes around the world, work for a day as in an international collaboration and publish the results.

In practice, the detector used is the CRC described in the previous paragraph, and the muon flux is measured as a function of the angle of arrival not uniformly distributed on the ground. By analyzing the arrival directions of these particles as a function of the angle they form with the local zenith, we find that the maximum flux occurs for $\theta=0^{\circ}$, i.e. for particles arriving perpendicular to the Earth's surface. The flux decreases continuously as the angle between the direction of incidence and the zenith increases. At $90^{\circ}$ the flux is minimal and the distribution of the measured values can be described using a $\cos ^{2}$ function. In the activity, questions and answers lead the reader to interpret the data as well as explain the experimental technique by reflecting on the geometry of the detector and introducing the basics of the types of measurement uncertainties.

\subsection{Measurement of muons as a function of height in the atmosphere}

The measurement described in this laboratory [10] was carried out during a balloon flight with the Stratospheric Balloon MoCRiS (Measurement of Cosmic Ray in Stratosphere) in 2019, that reached up to 35111 meters (Fig. 3a). The main aim of the launch was the measurement of the cosmic ray flux as a function of altitude, performed by two ArduSiPM particle detectors developed by the INFN Section of Rome (see the contribution by V. Bocci in this conference).

The MoCRiS experiment consists of a balloon and an octagonal shaped spacecraft containing several instruments partly replicated to ensure fault tolerance. The data collected by the following on-board instruments are used for the step-by-step analysis: GPS satellite navigation system, temperature and pressure sensor, two ArduSiPM particle detectors, two GoPRO cameras (video+photos) and dust capture. The path allows, by means of an Excel spreadsheet, for the data analysis, to verify the pressure-to-height relation, the height of the thermal inversion in the atmosphere, reaching and exceeding the maximum Regener-Pfotzer level of the shower development (Fig. 3b). 

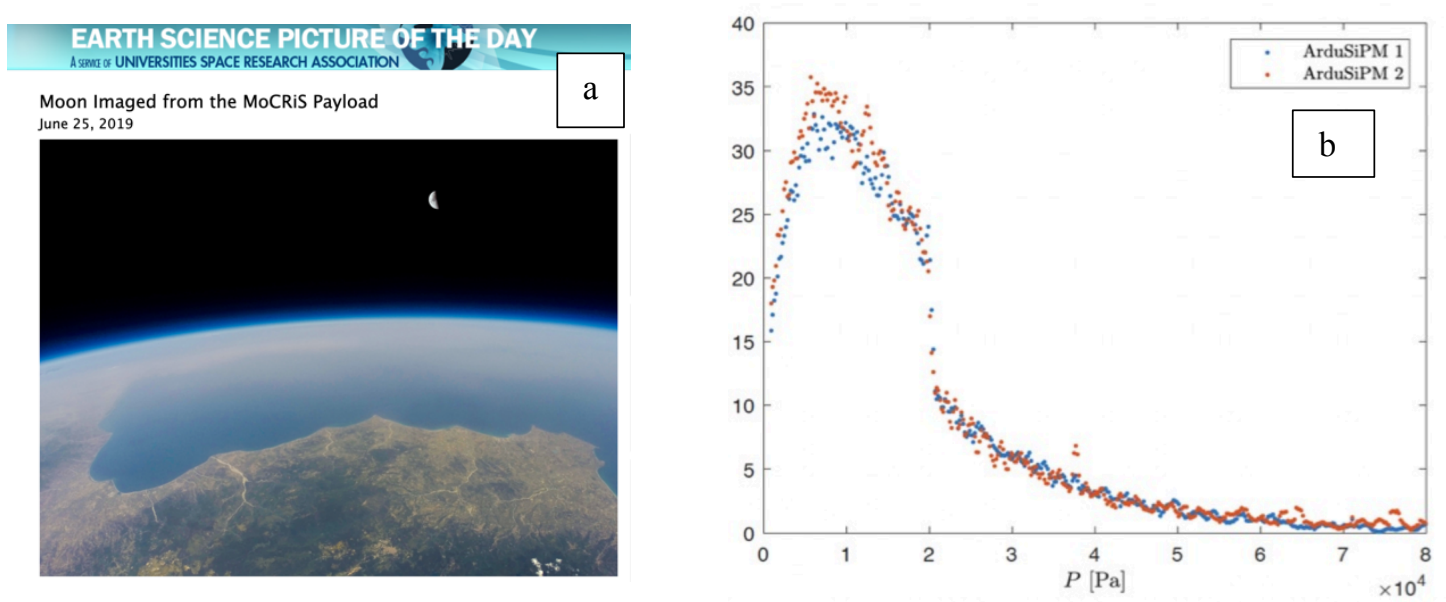

Figure 3: (a) Moon from MoCRiS Payload and (b) reconstruction of flux vs atmospheric pressure.

\subsection{Measurement of muons as a function of depth in water}

In this activity the measurement carried out by D. Pacini in 1911 of the flux of atmospheric muons in water, first in a reservoir and then in the open sea, at the military academy in Livorno, Italy, is reproduced [11]. Geiger counters equipped with a high-precision counter tube to detect alpha, beta and gamma rays are used (Fig. 4a). The data are stored in an internal memory, and can be read via a USB interface. In the activity, using an Excel spreadsheet, the decrease of events as a function of depth is verified (Fig. 4 b). The presence of opposing wind and current changed the fit at sea, the measurements at depths greater than $3 \mathrm{~m}$ (light points) were corrected for the effect of the angle to the vertical (dark points). From the fit of the experimental points with the function $h_{0} \cdot\left[1-f \cdot\left(1-e^{-x / h}\right)\right]$ we find a radiation fraction at the surface of about $f=0.26$ and an attenuation length of about $h=28 \mathrm{~cm}$, the $68 \%$ bands are also calculated.
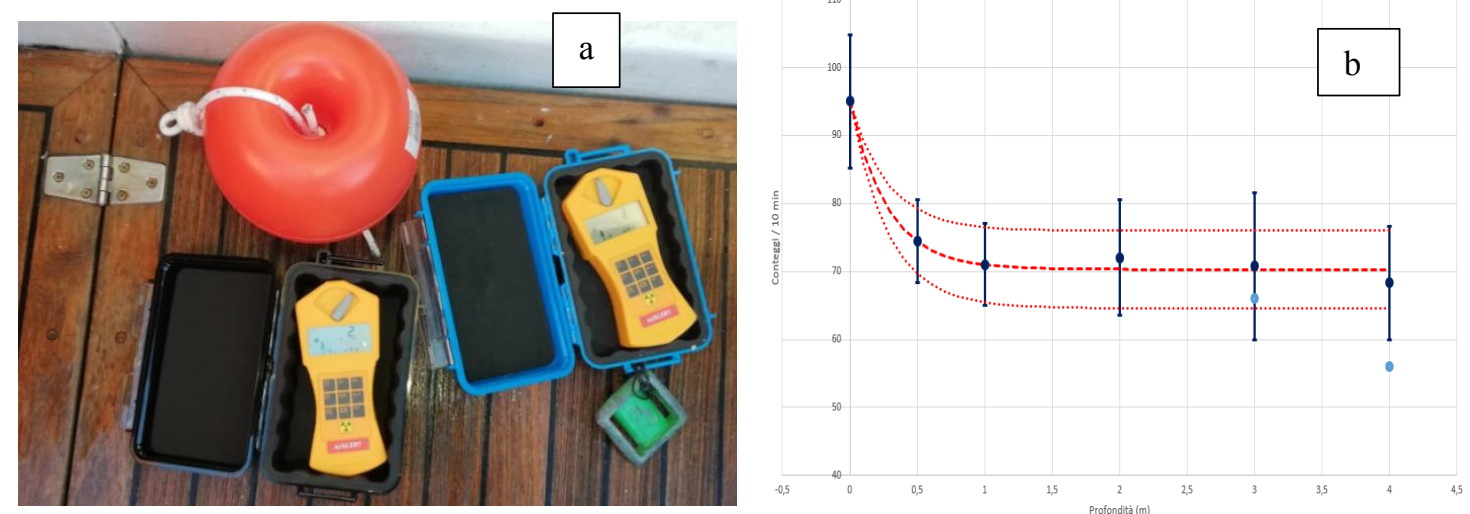

Figure 4: (a) Geiger counters and (b) decrease of events as a function of depth.

\subsection{Pierre Auger Observatory}

The Pierre Auger Observatory [4] is the world's largest observatory for the study of ultrahigh energy cosmic rays $\left(\mathrm{E}>10^{18} \mathrm{eV}\right)$. It is located in the Argentine pampas at $1400 \mathrm{~m}$ above sea level and covers an area of $3000 \mathrm{~km}^{2}$. The Observatory consists of two main detectors, the Surface Detector (SD) and the Fluorescence Detector (FD) optimized to study Extended Atmospheric Shower (EAS), i.e. the set of particles, called secondary cosmic rays, produced by the collision of primary cosmic rays with the nuclei of the Earth's atmosphere. The Observatory has made public $10 \%$ of the data acquired so far (Pierre Auger Collaboration 2021, Auger Open Data release 12021, DOI:10.5281/zenodo.4487613) (see the contribution by V. Scherini in this conference), which can be used to carry out simple data analysis exercises reported in the online pathway [12]. Each exercise is carried out using a worksheet (notebook) provided by using Google Colab and 
the Python programming language. Knowing the energy of the showers detected by the Observatory and the number of showers collected in a certain energy range, we can calculate the flux and construct the spectrum of primary cosmic rays (Fig. 5a). It is also possible to construct a galaxy map and search for excesses of very high energy cosmic rays (Fig. 5b), all in a very interactive way and with step-by-step explanations.
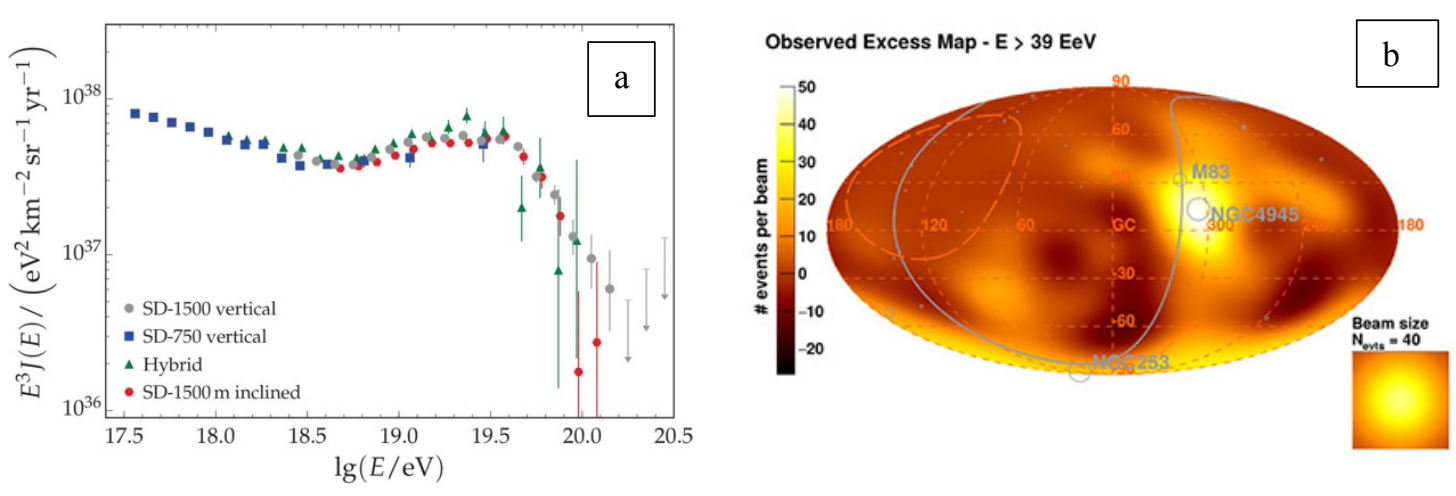

Figure 5: (a) Auger ultra-high energy cosmic rays' spectrum and (b) observed excess map with energy greater than 6 Joules. Ref. Pierre Auger Observatory www.auger.org

\subsection{Teachers' Area}

The activities on the OCRA website are completed by the space dedicated to the teachers [13], which begins with a brief introduction to the most common teaching methodologies, provides an overview on materials to support for the development of teaching projects in the classroom, with a broad overview of current legislation as well as indications of international platforms that deal with identifying the objectives in various school contexts, analyzing and monitoring the degree to which they are pursued. There are also indications on teaching activities in CLIL (Content and Language Integrated Learning) mode, as well as a handbook on teaching design.

\section{The OCRA online course for teachers}

The online laboratories described above were presented in an online meeting dedicated to high school teachers on the INFN Edu Physics YouTube channel and on the OCRA Facebook page [14] (Fig.6). More than 200 teachers took part in the online meeting in January and 70 of them enrolled in the 9-meeting course in spring 2021. The first two meetings dealt with an introduction to cosmic ray physics and a hands-on approach, then the laboratories available on the website were presented in a dedicated meeting. Each seminar was 2 hours long and after a short introduction, the teachers were able to engage in hands-on activities to analyze the data from each lab as well as ask questions and report on their experience in class. The aim of the course was to provide teachers with the manual skills of the tools, after introducing the phenomenological part, so that they can organize activities with students on these aspects in the classroom.

\section{Conclusion and future activities}

Since its foundation, OCRA has made a substantial and effective contribution to the implementation of the INFN's cosmic ray outreach activities, adding other activities that have developed in the meantime, also in response to specific needs, such as the online laboratories developed during the first spring lockdown. 


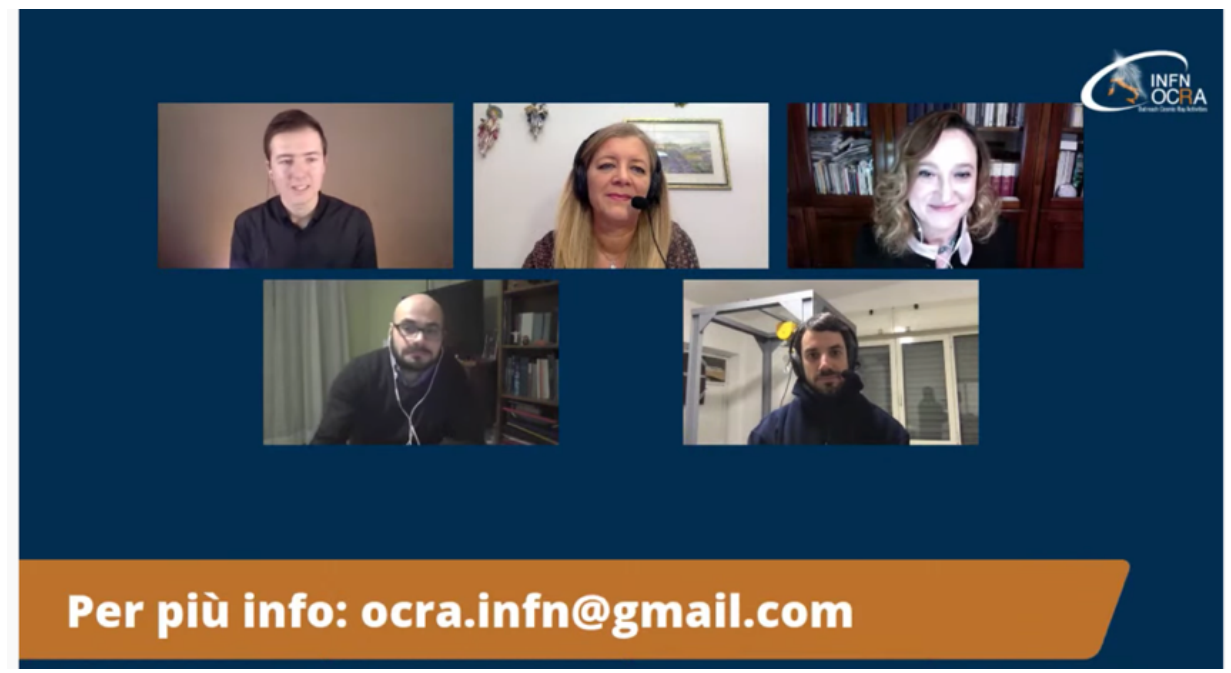

Figure 6: Image of the online teachers' course introduction event.

The fact that the initiative is heading in the right direction is demonstrated by the enthusiastic and interested participation of the teachers and students in the activities being carried out. In the future, the standard initiatives, such as the ICD and the science camp for students, will continue to be implemented, but others will also be developed, in particular teacher courses, both face-to-face and online, as well as masterclasses in high schools.

\section{References}

[1] 36th International Cosmic Ray Conference -ICRC2019- July 24th - August 1st, 2019 PoS(ICRC2019)173. Outreach Cosmic Ray Activities - OCRA. C. Aramo. S. Hemmer

[2] https://web.infn.it/OCRA/percorso-raggi-cosmici/

[3] https://www.youtube.com/watch?v=ZNoG2etdJ7s

[4] Pierre Auger Coll., Nucl.Instrum.Meth. A798 (2015) 172-213, https://www.auger.org/index.php

[5] https://web.infn.it/OCRA/misura-della-rate-di-muoni-cosmici/

[6] PoS EPS-HEP2017 (2018) 549 C. Aramo et al.: "Go to the astroparticle physics school with the Toledo Metro Station Totem-Telescope for cosmic rays"

[7] https://web.infn.it/OCRA/misura-della-distribuzione-angolare-dei-muoni/

[8] https://web.infn.it/OCRA/misura-dei-muoni-in-funzione-dellangolo-di-zenith/

[9] https://icd.desy.de/

[10] https://web.infn.it/OCRA/misura-dei-muoni-in-funzione-dellaltezza-in-atmosfera/

[11] https://web.infn.it/OCRA/misura-dei-muoni-in-funzione-della-profondita-in-acqua/

[12] https://web.infn.it/OCRA/losservatorio-pierre-auger/

[13] https://web.infn.it/OCRA/raccolta-di-materiale-per-docenti/

[14] https://youtu.be/ZNoG2etdJ7s, https://www.facebook.com/ocra.infn.1/posts/442461246949492 


\section{Full Authors List: OCRA Collaboration}

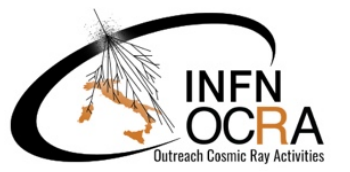

F. Alemanno ${ }^{1,2}$, R. Aloisio ${ }^{1,2}$, C. Altomare ${ }^{3,4}$, R. Antolini ${ }^{1}$, C. Aramo $^{5}$, C. Arcaro $^{6}$, F.C.T. Barbato ${ }^{1,2}$, M. Battaglieri ${ }^{7}$, M. Battisti ${ }^{8,9}$, A. Bau ${ }^{10,11}$, V.E. Bellinzona ${ }^{12}$, P. Bernardini ${ }^{13,14}$, A. Bersani ${ }^{7}$, M. $^{2}$ Bertaina $^{8,9}$, A. Berti8, B. Bertucci ${ }^{15,16}$, F. Bisconti ${ }^{8,9}$, E. Bissaldi ${ }^{3,4}$, V. Bocci ${ }^{17}$, M. Boezio ${ }^{18,19}$, D. Boncioli $^{1,20}$, M. Bondi ${ }^{7}$, L. Bonechi ${ }^{21}$, R. Bonino ${ }^{8,9}$, G. Bonnoli $^{22}$, V. Bonvicini ${ }^{18}$, E. Bossini ${ }^{22,23}$, B. Bottino $^{7,24}$, M. Buscemi $^{25}$, B. Caccianiga ${ }^{26}$, L. Caccianiga ${ }^{26}$, A. Candela ${ }^{1}$, A. Capone ${ }^{17,27}$, M. Cariello ${ }^{7}$,

R. Caruso ${ }^{25,28}$, G. Cataldi $^{13}$, G. Chiodi ${ }^{17}$, G. Chiodini $^{13}$, R. Colalillo ${ }^{5,29}$, M.R. Coluccia ${ }^{13}$, F. Convenga $^{13,14}$, S. Copello ${ }^{7,30}$, M. Corosu ${ }^{7}$, D. D’Urso ${ }^{31,32}$, F. Dal Corso ${ }^{6}$, S. Davini ${ }^{7}$, M. De Deo ${ }^{1}$, S. De Gateano ${ }^{3,4}$, M. De Laurentis ${ }^{5,29}$, I. De Mitri ${ }^{1,2}$, F. De Palma ${ }^{13,14}$, E. De Vito ${ }^{13,14}$, D. Dell'Aquila ${ }^{31,32}$, D. Depaoli ${ }^{8,9}$, A. Di Luca ${ }^{12}$, F. Di Pierro ${ }^{8}$, B. Di Ruzza ${ }^{12}$, M. Di

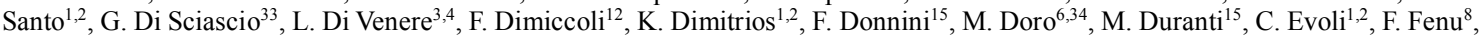
F. Fontanelli $i^{7,30}$, P. Fusco ${ }^{3,4}$, F. Gargano ${ }^{3}$, M. Gervasi ${ }^{10,11}$, A. Giampaoli ${ }^{1}$, N. Giglietto ${ }^{3,4}$, F. Giordano ${ }^{3,4}$, I. Gnesi ${ }^{35,36}$, S. Gonzi ${ }^{21}$, D. Grandi $^{10,11}$, M. Graziani ${ }^{15,16}$, S. Hemmer ${ }^{6}$, F. Iacoangeli $i^{17}$, A. Insolia ${ }^{25,28}$, S. Iovenitti ${ }^{26,37}$, V. Ippolito ${ }^{17}$, G. La Vacca ${ }^{10,11}$, G. La Verde ${ }^{5,29}$, E. Leonora $^{25}$, S. Levorato ${ }^{18}$, D. Liguori ${ }^{35,38}$, P. Lipari ${ }^{17}$, F. Longo ${ }^{18,39}$, F. Loparco ${ }^{3,4}$, R. Lopez Coto $^{6}$, S. Loporchio ${ }^{3}$, A. Marino ${ }^{5,40}$, G. Marsella $^{25,41}$, D. Martello ${ }^{13,14}$, M.N. Mazziotta ${ }^{3}$,A. Menegollii ${ }^{42,43}$, S. Miozzi ${ }^{33}$, H. Miyamoto ${ }^{8}$, E. Mocchiutti ${ }^{18}$, S. Morganti ${ }^{17}$, F. Morsani $^{22}$, R. Munini ${ }^{18,19}$, R. Mussa ${ }^{8}$, F. Nozzoli ${ }^{12}$, A. Nucita ${ }^{13,14}$, G.C. Organtini ${ }^{17,27}$, G. Ottonello ${ }^{7}$, F. Pantaleo ${ }^{3,4}$, R. Paoletti ${ }^{22}$, F. Parodi $^{7}$, L. Perrone ${ }^{13,14}$, L. Pesenti ${ }^{10,11}$, S. Petrera ${ }^{1,2}$, C. Petronio ${ }^{35,44}$, R. Pillera ${ }^{3,4}$, F. Pilo ${ }^{22}$, C. Pizzolotto ${ }^{18}$, E. Prandini ${ }^{6,34}$, M. Pugliese $^{5,29}$, S. Raino $\dot{1}^{3,4}$, N. Randazzo ${ }^{25}$, R. Rando ${ }^{6,34,45}$, L. Recchia ${ }^{17}$, E. Ricci ${ }^{12}$, M. Rinaudo ${ }^{8,9}$, V. Rizi $^{1,20}$, N. Rossi ${ }^{1}$, D. Rozza ${ }^{31,32}$, F. Salamida $^{1,20}$, P. Savina ${ }^{13,14}$, V. Scherini ${ }^{13,14}$, M. Schioppa ${ }^{35,46}$, V. Scotti ${ }^{5,29}$, D. Serini ${ }^{3}$, V. Sipala ${ }^{31,32}$, A. Surdo ${ }^{13,14}$, A. Tiberio ${ }^{21}, N$. Tomassetti $^{15,16}$, C. Tomei ${ }^{17}$, I. Tosta E Melo ${ }^{11,32}$, P. Turco ${ }^{35,46}$, E. Vannuccini ${ }^{21}$, V. Vecchiotti ${ }^{1,2}$, I. Veronesi ${ }^{5,47}$, G. Zampa ${ }^{18}$

${ }^{1}$ INFN Laboratori Nazionali del Gran Sasso, Assergi, Italy

${ }^{2}$ Gran Sasso Science Institute, L’Aquila, Italy

${ }^{3}$ INFN Sezione di Bari, Bari, Italy

${ }^{4}$ Dipartimento Interateneo di Fisica "M. Merlin", University and Politecnico of Bari, Bari, Italy

${ }^{5}$ INFN Sezione di Napoli, Naples, Italy

${ }^{6}$ INFN Sezione di Padova, Padua, Italy

${ }^{7}$ INFN Sezione di Genova, Genua, Italy

${ }^{8}$ INFN Sezione di Torino, Turin, Italy

${ }^{9}$ Dipartimento di Fisica, University of Turin, Turin, Italy

${ }^{10}$ INFN Sezione di Milano Bicocca, Milan, Italy

${ }^{11}$ Dipartimento di Fisica "Giuseppe Occhialini", University of Milan-Bicocca, Milan, Italy

${ }^{12}$ Trento Institute for Fundamental Physics and Applications, Trento, Italy

${ }^{13}$ INFN Sezione di Lecce, Lecce, Italy

${ }^{14}$ Dipartimento di Matematica e Fisica “Ennio de Giorgi”, University of Salento, Lecce, Italy

${ }^{15}$ INFN Sezione di Perugia, Perugia, Italy

${ }^{16}$ Dipartimento di Fisica e Geologia, University of Perugia, Perugia, Italy

${ }^{17}$ INFN Sezione di Roma I, Rome, Italy

${ }^{18}$ INFN Sezione di Trieste, Trieste, Italy

${ }^{19}$ Institute for Fundamental Physics of the Universe, Trieste, Italy

${ }^{20}$ Dipartimento di Scienze Fisiche e Chimiche, University of L'Aquila, L'Aquila, Italy

${ }^{21}$ INFN Sezione di Firenze, Florence, Italy

${ }^{22}$ INFN Sezione di Pisa, Pisa, Italy

${ }^{23}$ Dipartimento di Fisica, University of Pisa, Pisa, Italy

${ }^{24}$ Physics Department, Princeton University, Princeton, USA

${ }^{25}$ INFN Sezione di Catania, Catania, Italy 
${ }^{26}$ INFN Sezione di Milano, Milan, Italy

${ }^{27}$ Dipartimento di Fisica, University La Sapienza, Rome, Italy

${ }^{28}$ Dipartimento di Fisica e Astronomia “E. Majorana”, University of Catania, Catania, Italy

${ }^{29}$ Dipartimento di Fisica "Ettore Pancini”, University of Naples "Federico II", Naples, Italy

${ }^{30}$ Dipartimento di Fisica, University of Genua, Genua, Italy

${ }^{31}$ INFN Laboratori Nazionali del Sud, Catania, Italy

${ }^{32}$ Dipartimento di Chimica e Farmacia, University of Sassari, Sassari, Italy

${ }^{33}$ INFN Sezione di Roma Tor Vergata, Roma, Italy

${ }^{34}$ Dipartimento di Fisica e Astronomia “G. Galilei”, University of Padua, Padua, Italy

${ }^{35}$ INFN Laboratori Nazionali di Frascati, Gruppo Collegato di Cosenza, Cosenza, Italy

${ }^{36}$ Centro Ricerche Enrico Fermi, Rome, Italy

${ }^{37}$ Dipartimento di Fisica “A. Pontremoli”, University of Milan, Milan, Italy

${ }^{38}$ Liceo Patrizi, Cariati, Italy

${ }^{39}$ Dipartimento di Fisica, University of Trieste, Trieste, Italy

${ }^{40} \mathrm{CNR}$ - Istituto di Scienze Applicate e Sistemi Intelligenti “Eduardo Caianiello”, Pozzuoli, Italy

${ }^{41}$ Dipartimento di Fisica e Chimica “Emilio Segrè”, University of Palermo, Palermo, Italy

${ }^{42}$ INFN Sezione di Pavia, Pavia, Italy

${ }^{43}$ Dipartimento di Fisica, University of Pavia, Pavia, Italy

${ }^{44}$ Liceo Volta, Reggio Calabria, Italy

${ }^{45}$ Centro di Ateneo di Studi e Attività Spaziali “G. Colombo”, University of Padua, Padua, Italy

${ }^{46}$ Dipartimento di Fisica, University of Calabria, Arcavacata di Rende, Italy

${ }^{47}$ Dipartimento di Matematica, University of Salerno, Salerno, Italy 